$\mathrm{ITP}-\mathrm{UH}-07 / 98$

hep-th/9804153

\title{
Deformations of global symmetries in the extended antifield formalism
}

\author{
Friedemann Brandt \\ Institut für Theoretische Physik, Universität Hannover, \\ Appelstraße 2, D-30167 Hannover, Germany \\ E-mail: brandt@itp.uni-hannover.de
}

\begin{abstract}
It is outlined how deformations of field theoretical rigid symmetries can be constructed and classified by cohomological means in the extended antifield formalism. Special attention is devoted to deformations referring only to a subset of the rigid symmetries of a given model and leading to a nontrivial extension of the graded Lie algebra associated with that subset. The method is illustrated for a $\mathrm{D}=4, \mathrm{~N}=2$ supersymmetric model where the central extension of the supersymmetry algebra emerges via a deformation. Deformations of gauge fixed actions with a BRST symmetry are discussed too and illustrated by the Curci-Ferrari model.
\end{abstract}

\section{Introduction}

A problem often met in field theory is to what degree a given action functional can be nontrivially deformed while keeping some of its symmetries. A particularly interesting issue is whether the symmetry transformations themselves can be deformed in a nontrivial way, i.e. whether there are simultaneous deformations of the action and its symmetries.

Deformations of this sort can be studied systematically by cohomological methods in the spirit of Gerstenhaber's approach to deformation theory [1]. This was first described in [2] (see also [3, 4] ) for gauge symmetries in the framework of the standard antifield formalism [5, 6, 7]. The inclusion of rigid (= global) symmetries was roughly sketched more recently in [8] within an extended antifield formalism. The aim of this work is to develop the latter approach more thoroughly, with special attention to deformations which are required to maintain only (a deformed version of) a subset of the rigid symmetries of a given model.

The restriction to a subset of the rigid symmetries is a typical situation, as often it is neither possible nor desirable to keep all the rigid symmetries when deforming a field theory because that may constrain the sought deformations too much. We shall thus base the deformation theory on an extended antifield formalism which involves only a "closed" subset of rigid symmetries. The "closure" of the subset requires 
that the graded commutator algebra of the rigid symmetries under study closes in the soft (field theoretical) sense, i.e. up to gauge transformations and on-shell trivial symmetries . In other words, a closed subset of rigid symmetries forms a subalgebra (in the soft sense) of the graded commutator algebra of all the rigid symmetries.

When one applies the extended antifield formalism to study deformations of such a subset of rigid symmetries, one may encounter a "subtlety". Namely, a deformation may turn a subset of rigid symmetries which is closed in the soft sense into an open one. That is, it can happen that the deformed commutator algebra involves symmetries which did not occur in the undeformed one. These additional symmetries are not "new" ones which are introduced through the deformation. Rather, they are present already in the original (undeformed) model. The subtlety is that usually it is not clear from the outset which additional symmetries of the original model can show up in the deformed commutator algebra. In particular, this may depend on the deformation itself.

Hence, the property of a subset of rigid symmetries to be a closed one is not necessarily preserved by deformations. This is actually an interesting phenomenon as it is related to extensions of the (graded) Lie algebra associated with the commutator algebra of the subset of rigid symmetries under study. Important examples are central extensions of extended supersymmetry algebras [10]. As an illustration, we shall discuss a simple four dimensional $\mathrm{N}=2$ supersymmetric model for a hypermultiplet [11, 12] where the central extension of the supersymmetry algebra arises indeed via a deformation of the model.

The antifield formalism serves in this context as a tool that allows one to formulate the deformation theory conveniently in cohomological terms. Ghost fields are not dynamical in this approach (in particular, they are not paired with antighost fields), in contrast to their counterparts in the quantum field theoretical context. Nevertheless, the formalism applies also to gauge fixed action functionals which contain dynamical ghost and antighost fields. This application just requires a slight change of the point of view as compared to the one familiar from quantum field theory. Namely, the gauge fixed action simply takes the role of a classical action. Accordingly, the dynamical ghost and antighost fields occurring in the gauge fixed action are counted among the classical fields, and the BRST symmetry of the gauge fixed action counts among the rigid symmetries. In particular this allows one to investigate deformations of the BRST symmetry after fixing the gauge. We shall discuss and illustrate this particular application in some detail in the Curci-Ferrari model [13, 14, 15].

The paper has been organized as follows. Section प 1 summarizes basic properties of global and local symmetries in Lagrangian field theory which are used later on. Then the extended antifield formalism and the construction and properties of the extended BRST differential are briefly reviewed in sections [II and IV. The systematic approach to the deformation problem is described in section $\square$. Sections VI and VII contain the examples mentioned above, i.e. the hypermultiplet of $\mathrm{N}=2$ supersymmetry and

\footnotetext{
${ }^{1}$ In order to set up the extended antifield formalism, it may be necessary to include also "symmetries of higher order" [8, 9].
} 
the Curci-Ferrari model. The paper is ended with some concluding remarks in section VIII.

\section{Global and local symmetries}

We shall first briefly summarize the definition and some properties of continuous rigid and gauge symmetries in Lagrangian field theories, following the presumably most popular approach based on the action (alternatively one can define rigid and gauge symmetries on the level of the field equations, via conserved currents and Noether identities respectively). We shall thus consider Lagrangian field theories which derive from an action functional for a set of fields $\phi^{i}(x)$,

$$
S_{\text {class }}[\phi]=\int d^{n} x L(x,[\phi])
$$

where $L(x,[\phi])$ is a Lagrangian constructed of the fields and their partial derivativeset. The field equations (equations of motion) derive via the variational principle from $S_{\text {class }}$, i.e. they are the corresponding Euler-Lagrange equations.

A continuous rigid symmetry of an action (2.1) is generated by transformations of the fields with a constant infinitesimal parameter $\varepsilon$,

$$
\phi^{i} \rightarrow \tilde{\phi}^{i}=\phi^{i}+\varepsilon G^{i}(x,[\phi]), \quad \varepsilon=\text { constant }
$$

such that $L(x,[\tilde{\phi}])$ differs from $L(x,[\phi])$ to first order in $\varepsilon$ at most by a total derivative,

$$
L(x,[\tilde{\phi}])=L(x,[\phi])+\varepsilon \partial_{\mu} k^{\mu}(x,[\phi])+O\left(\varepsilon^{2}\right) .
$$

A gauge symmetry of an action (2.1) is defined similarly, with the important difference that it involves, instead of a constant parameter, an additional field $\lambda=\lambda(x)$ (i.e. a field which does not occur in the Lagrangian). It is generated by infinitesimal transformations of the form

$$
\phi^{i} \rightarrow \tilde{\phi}^{i}=\phi^{i}+\sum_{k \geq 0} r^{i \mu_{1} \ldots \mu_{k}}(x,[\phi]) \partial_{\mu_{1}} \ldots \partial_{\mu_{k}} \lambda
$$

such that $L(x,[\tilde{\phi}])$ and $L(x,[\phi])$ differ to first order in $\lambda$ at most by a total derivative,

$$
L(x,[\tilde{\phi}])=L(x,[\phi])+\partial_{\mu} h^{\mu}(x,[\phi, \lambda])+O\left(\lambda^{2}\right) .
$$

This invariance condition must hold for an unconstrained field $\lambda$, i.e., it must neither impose a differential equation for $\lambda$, nor determine $\lambda$ in terms of the fields $\phi^{i}$ and their

\footnotetext{
${ }^{2}$ Here and in the following, $[\phi]$ denotes collectively dependence on the fields and on their derivatives. In more precise mathematical terms, $\phi^{i}, \partial_{\mu} \phi^{i}, \partial_{\mu} \partial_{\nu} \phi^{i}, \ldots$ are to be understood as local coordinates of a jet space, and $\phi^{i}(x)$ as sections of the jet bundle over an $n$-dimensional base manifold ("spacetime") with local coordinates $x^{\mu}(\mu=1, \ldots, n)$. The arguments of $L(x,[\phi])$ indicate that the Lagrangian may (but, of course, need not) depend explicitly on the $x^{\mu}$.
} 
derivatives (otherwise $\lambda$ would turn into a function of the $x^{\mu}, \phi^{i}$ and their derivatives and thus (2.4) would reduce to a rigid symmetry of the form (2.2)).

Now, the above standard definitions do not yet characterize symmetries satisfactorily for our purpose. An important ingredient, underplayed in many textbooks, is still missing: the distinction between trivial and nontrivial symmetries. For instance, consider transformations (2.2) and (2.4) with

$$
\begin{aligned}
& G^{i}(x,[\phi])=E^{i j}(x,[\phi]) \frac{\hat{\partial} L}{\hat{\partial} \phi^{j}} \\
& \sum_{k \geq 0} r^{i \mu_{1} \ldots \mu_{k}}(x,[\phi]) \partial_{\mu_{1}} \ldots \partial_{\mu_{k}} \lambda=E^{i j}(x,[\phi, \lambda]) \frac{\hat{\partial} L}{\hat{\partial} \phi^{j}}
\end{aligned}
$$

where $\hat{\partial} L / \hat{\partial} \phi^{i}$ is the Euler-Lagrange derivative of the Lagrangian with respect to $\phi^{i}$,

$$
\frac{\hat{\partial} L}{\hat{\partial} \phi^{i}}=\frac{\partial L}{\partial \phi^{i}}-\partial_{\mu} \frac{\partial L}{\partial\left(\partial_{\mu} \phi^{i}\right)}+\ldots
$$

and $E^{i j}$ are any functions which are only required to be graded antisymmetric in their indices,

$$
E^{i j}=-(-)^{\epsilon_{i} \epsilon_{j}} E^{j i},
$$

where $\epsilon_{i}$ is the Grassmann parity of $\phi^{i}$. It is easily verified that (2.6) and (2.7) give rigid and gauge symmetries, satisfying (2.3) and (2.5) respectively, for any choice of $E^{i j}$ fulfilling (2.9). Such symmetries are examples of trivial symmetries which may be called "on-shell trivial symmetries" (the terminology reflects that the symmetry transformations vanish for every solution of the field equations, as the latter read $\left.\hat{\partial} L / \hat{\partial} \phi^{i}=0\right)$. More general trivial symmetries of this type are obtained from (2.6) and (2.7) when $E^{i j}$ are differential operators of the form $E^{i j}=\sum e^{i j \mu_{1} \ldots \mu_{k}} \partial_{\mu_{1}} \ldots \partial_{\mu_{k}}$ with properties generalizing (2.9) appropriately.

In addition to on-shell trivial symmetries, there is a second type of trivial rigid symmetries whenever the action possesses a true gauge symmetry, i.e. a gauge symmetry which is not on-shell trivial. Indeed, in that case the action has automatically infinitely many further rigid symmetries which are to be considered as trivial too, even though they are not on-shell trivial. These additional trivial rigid symmetries arise from nontrivial gauge transformations (2.4) by replacing there $\lambda$ with $\varepsilon f(x,[\phi])$, where $f(x,[\phi])$ is any function of the fields and their derivatives. Indeed, as (2.5) holds for any $\lambda$, such a replacement results in a transformation (2.2) satisfying (2.3) with

$$
G^{i}(x,[\phi])=\sum_{k \geq 0} r^{i \mu_{1} \ldots \mu_{k}}(x,[\phi]) \partial_{\mu_{1}} \ldots \partial_{\mu_{k}} f(x,[\phi]) .
$$

Hence, every action has infinitely many trivial gauge and rigid symmetries. Gauge and rigid symmetries are therefore best defined as equivalence classes where two symmetries are called equivalent when they differ by a trivial symmetry (or by irrelevant 
redefinitions of the respective $\varepsilon$ and $\lambda$, i.e. by multiplications of $\varepsilon$ and $\lambda$ with arbitrary constants and field dependent functions respectively $\left.{ }^{3}\right)$. One can then introduce the concept of a basis of symmetries, containing one representative of each nontrivial equivalence class. We shall characterize such bases for the gauge and rigid symmetries through operations $\left\{\delta_{\alpha}\right\}$ and $\left\{\Delta_{a}\right\}$ respectively, which are related to symmetry transformations (2.2) and (2.4) according to

$$
\begin{aligned}
\delta_{\alpha} \phi^{i} & =R_{\alpha}^{i}(x,[\phi]) \equiv \sum_{k \geq 0} r_{\alpha}^{i \mu_{1} \ldots \mu_{k}}(x,[\phi]) \partial_{\mu_{1}} \ldots \partial_{\mu_{k}} \\
\Delta_{a} \phi^{i} & =G_{a}^{i}(x,[\phi]) .
\end{aligned}
$$

As the graded commutator of two infinitesimal symmetry transformations is automatically again an infinitesimal symmetry transformation (due to the derivation property of infinitesimal transformations), there is always a graded commutator algebra associated with such bases. However, due to the presence of trivial symmetries, this graded commutator algebra is in general a quotient algebra because, in general, the graded commutator of two elements of the basis can be expressed in terms of elements of the same basis only up to trivial symmetries. In particular, the general form of the graded commutator of any two elements of a basis of infinitesimal rigid symmetry transformations is thus

$$
\left[\Delta_{a}, \Delta_{b}\right] \phi^{i}=f_{a b}^{c} \Delta_{c} \phi^{i}+R_{\alpha}^{i} f_{a b}^{\alpha}(x,[\phi])+E_{a b}^{i j}(x,[\phi]) \frac{\hat{\partial} L}{\hat{\partial} \phi^{j}}
$$

where the graded commutator of two objects $A$ and $B$ is defined by means of their Grassmann parities $\epsilon(A)$ and $\epsilon(B)$ through

$$
[A, B]=A B-(-)^{\epsilon(A) \epsilon(B)} B A \text {. }
$$

In (2.13), $f_{a b}^{c}$ are constant coefficients which are the structure constants of a graded Lie algebra (as a consequence of $\left[\left[\Delta_{a}, \Delta_{b}\right], \Delta_{c}\right]+$ cyclic $=0$ ), while $f_{a b}^{\alpha}(x,[\phi])$ and $E_{a b}^{i j}(x,[\phi])$ are in general field dependent functions and operators appearing in trivial rigid symmetries as described above, cf. (2.10) and (2.6).

\section{Extended antifield formalism}

We shall now recall the basic features of the extended antifield formalism and fix our notation and conventions. For simplicity, we shall concentrate on the case that the gauge transformations (if any) are irreducible and that only ordinary rigid symmetries are present or needed, but no rigid symmetries of higher order in the terminology of [8]. The general case is a straightforward extension of this one. As mentioned already, the extended antifield formalism can be established for any closed subset of

\footnotetext{
${ }^{3}$ Clearly, two symmetries differing only through such redefinitions are to be identitied, as (2.3) and (2.5) must hold for arbitrary constant parameters $\varepsilon$ and unconstrained fields $\lambda$.
} 
rigid symmetries [8]. When higher order rigid symmetries are absent, a closed subset is simply a subset $\left\{\Delta_{\underline{a}}\right\}$ of a basis $\left\{\Delta_{a}\right\}$ of nontrivial rigid symmetries such that, in the notation of the previous section,

$$
\left\{\Delta_{a}\right\}=\left\{\Delta_{\underline{a}}, \Delta_{\hat{a}}\right\} \quad, \quad f_{\underline{a b}}^{\hat{c}}=0 \quad \forall \underline{a}, \underline{b}, \hat{c} .
$$

$f_{a b}^{\hat{c}}=0$ requires that the graded commutator algebra of the $\Delta_{\underline{a}}$ is a subalgebra of (2.13) in the "soft" sense, i.e. with respect to the quotient structure "modulo trivial rigid symmetries".

The fields and antifields of the standard antifield formalism are denoted by $\Phi^{A}$ and $\Phi_{A}^{*}$ where $\left\{\Phi^{A}\right\}$ contains the "classical" fields $\phi^{i}$, i.e. the fields occurring in the "classical" action (2.1) under study, and the ghost fields $C^{\alpha}$ corresponding to the nontrivial gauge symmetries of this action' The extended antifield formalism, restricted to the subset $\left\{\Delta_{\underline{a}}\right\}$, contains in addition a constant ("global") ghost $\xi \underline{a}$ for each $\Delta_{\underline{a}}$. These global ghosts have ghost number 1 and Grassmann parity opposite to the corresponding rigid symmetries. It is also very convenient (though not necessary in principle) to accompany each $\xi^{\underline{a}}$ with a constant antifield $\xi_{a}^{*}$. The latter has ghost number $(-2)$ and Grassmann parity opposite to $\xi \underline{a}$. In particular this allows one to set up the extended antifield formalism through an extended master equation of the form

$$
(S, S)=0
$$

where $($, ) is an extended antibracket defined by

$$
\begin{aligned}
(X, Y)= & \frac{\partial^{R} X}{\partial \xi \underline{a}} \frac{\partial^{L} Y}{\partial \xi_{\underline{a}}^{*}}-\frac{\partial^{R} X}{\partial \xi_{\underline{a}}^{*}} \frac{\partial^{L} Y}{\partial \xi \underline{a}} \\
& +\int d^{n} x\left[\frac{\delta^{R} X}{\delta \Phi^{A}(x)} \frac{\delta^{L} Y}{\delta \Phi_{A}^{*}(x)}-\frac{\delta^{R} X}{\delta \Phi_{A}^{*}(x)} \frac{\delta^{L} Y}{\delta \Phi^{A}(x)}\right] .
\end{aligned}
$$

Here superscripts $R$ and $L$ indicate right and left derivatives respectively. The extended antibracket is defined in the space of local functionals of the form

$$
\Gamma\left[\Phi, \Phi^{*}, \xi\right]+M^{\underline{a}}(\xi) \xi_{\underline{a}}^{*}
$$

where $\Gamma\left[\Phi, \Phi^{*}, \xi\right]$ is the spacetime integral of a local function of the fields and antifields which may depend on the global ghosts but not on the global antifields, and $M^{a}(\xi)$ is a polynomial in the global ghosts (note: $M \underline{a}(\xi) \xi_{\underline{a}}^{*}$ does not involve a spacetime integration). The solution $S$ of the extended master equation is a functional with ghost number 0 of the form (3.4). It contains the classical action, and encodes its gauge symmetries and the subset $\left\{\Delta_{a}\right\}$ of its rigid symmetries, as well as the graded commutator algebra of these symmetries. In addition one often imposes that $S$ be real. One then needs consistent conventions for complex conjugation. We denote

\footnotetext{
${ }^{4}$ As mentioned in the introduction, the "classical" action may be actually a gauged fixed one. Ghost and antighost fields occurring in such an action count among the $\phi^{i}$ and must not be confused with the $C^{\alpha}$, see section VII for an example.
} 
complex conjugation by a bar, and use the convention (familiar from supersymmetry, see, e.g., [16]) that complex conjugation of products involves a sign factor depending on the Grassmann parities,

$$
\overline{(X Y)}=(-)^{\epsilon_{X} \epsilon_{Y}} \bar{X} \bar{Y}
$$

The complex conjugate of an antifield $\Phi^{*}$ equals minus the antifield of the complex conjugate of $\Phi$ (independently of the Grassmann parity of $\Phi$ ),

$$
\overline{\left(\Phi^{*}\right)}=-(\bar{\Phi})^{*} \quad \forall \Phi \in\left\{\Phi^{A}, \xi^{\underline{a}}\right\} .
$$

For instance, with these conventions, the antifield of a real field is purely imaginary.

To describe and compute $S$, it is useful to expand it in the antifield number (agh). The latter vanishes for the fields, and equals minus the ghost number for the antifields,

$$
\begin{gathered}
\operatorname{agh} \phi^{i}=\operatorname{agh} C^{\alpha}=\operatorname{agh} \xi^{\underline{a}}=0 \\
\operatorname{agh} \phi_{i}^{*}=1, \quad \operatorname{agh} C_{\alpha}^{*}=\operatorname{agh} \xi_{\underline{a}}^{*}=2 .
\end{gathered}
$$

The expansion of $S$ is denoted by

$$
S=\sum_{k \geq 0} S_{k}, \quad \operatorname{agh} S_{k}=k
$$

Here $S_{0}$ is the classical action,

$$
S_{0}=S_{\text {class }}[\phi]
$$

$S_{1}$ encodes both the gauge transformations and the subset of the rigid symmetries under study,

$$
S_{1}=-\int d^{n} x\left(R_{\alpha}^{i} C^{\alpha}+\xi^{\underline{a}} \Delta_{\underline{a}} \phi^{i}\right) \phi_{i}^{*}
$$

where we used the notation of the previous section. $S_{2}$ encodes the graded commutator algebra of the gauge symmetries and the subset of rigid symmetries under study, and thus in particular the subalgebra of (2.13) referring to $\left\{\Delta_{\underline{a}}\right\}$,

$$
S_{2}=\frac{1}{2} \xi^{\underline{b}} \xi^{\underline{a}} \tilde{f}_{\underline{a} \underline{\underline{b}}}^{\underline{c}} \xi_{\underline{c}}^{*}+\int d^{n} x \xi^{\underline{b}} \xi^{\underline{a}}\left(\frac{1}{2} \tilde{f}_{\underline{a b}}^{\alpha} C_{\alpha}^{*}+\frac{1}{4} \phi_{i}^{*} \tilde{E}_{\underline{a b}}^{i j} \phi_{j}^{*}+\ldots\right),
$$

where $\tilde{f}_{\underline{a} \underline{b}}^{\underline{c}}, \tilde{f}_{\underline{a b}}^{\alpha}, \tilde{E}_{\underline{a b}}^{i j}$ coincide with $f_{\underline{a} \underline{\underline{b}}}^{\underline{c}}, f_{\underline{a b}}^{\alpha}, E_{\underline{a b}}^{i j}$ in (2.13) up to signs which follow from the formulae (e.g., $f_{\underline{a} \underline{b}}^{\frac{c}{b}}=(-)^{\epsilon_{b}}+1 f_{\underline{a} \underline{b}}^{c}$ where $\epsilon_{\underline{b}}$ is the Grassmann parity of $\Delta_{\underline{b}}$ ). The non-written terms in (3.11) encode analogously the graded commutator algebra of the gauge symmetries, and of the gauge symmetries with the $\Delta_{a}$. Higher terms $S_{k}$ $(k>2)$ in the expansion of $S$ reflect consistency relations following from the graded commutator algebra. The solution of the extended master equation encodes thus 
the complete algebraic structure of the gauge and rigid symmetries under study. In particular, the piece in $(S, S)=0$ which is linear in $\xi^{*}$ yields

$$
f_{[\underline{\underline{e} b} \underline{b}} f \frac{d}{\underline{c}] \underline{e}}=0
$$

where [...] indicates graded antisymmetrization. (3.12) is the Jacobi identity for the structure constants of a graded Lie algebra and reflects again that the commutator algebra of the $\Delta_{\underline{a}}$ constitutes a subalgebra of (2.13) in the soft sense. Of course, in general this commutator algebra is not a true graded Lie algebra, but still a graded Lie algebra in the soft sense.

\section{Extended BRST and Koszul-Tate differential}

The extended antifield formalism outlined in the previous section implies the existence of a nilpotent antiderivation which generalizes the standard BRST differential so as to incorporate rigid symmetries. We shall call this antiderivation the extended BRST differential and denote it by $s$. It is defined in the space of local functionals of the form (3.4) via the extended antibracket through

$$
s X=(S, X) .
$$

With this definition, $s$ squares to zero (= is "nilpotent"),

$$
s(X Y)=(s X) Y+(-)^{\epsilon_{X}} X(s Y), \quad s^{2}=0 .
$$

Furthermore, $s$ is a real differential if $S$ is a real functional. As $s$ is Grassmann odd, this means, due to (3.5),

$$
\overline{(s X)}=(-)^{\epsilon_{X}} s \bar{X} .
$$

It is useful to expand $s$ in the antifield number. The structure of $S$ implies that the expansion of $s$ starts with a piece $\delta$ that has antifield number -1 (i.e., $\delta$ lowers the antifield number by one unit),

$$
s=\delta+\gamma+\sum_{i \geq 1} s_{i}, \quad \operatorname{agh} \delta=-1, \operatorname{agh} \gamma=0, \operatorname{agh} s_{i}=i .
$$

The nilpotency of $s$ implies anticommutation relations between the pieces in this decomposition,

$$
\delta^{2}=0, \quad[\delta, \gamma]=0, \quad \gamma^{2}+\left[\delta, s_{1}\right]=0, \quad \ldots \quad .
$$

$\delta$ is the extension of the field theoretical Koszul-Tate differential [17, 18, 6]. It acts nontrivially only on the antifields, and coincides on $\Phi_{A}^{*}$ with the standard Koszul-Tate differential, while $\delta \xi_{\underline{a}}^{*}$ is an integrated local functional associated with the corresponding rigid symmetry,

$$
\begin{aligned}
& \delta \Phi^{A}=\delta \xi^{\underline{a}}=0, \quad \delta \phi_{i}^{*}=\frac{\hat{\partial}^{R} L}{\hat{\partial} \phi^{i}} \\
& \delta C_{\alpha}^{*}=R_{\alpha}^{i \dagger} \phi_{i}^{*}, \quad \delta \xi_{\underline{a}}^{*}=(-)^{\epsilon_{a}} \int d^{n} x\left(\Delta_{\underline{a}} \phi^{i}\right) \phi_{i}^{*}
\end{aligned}
$$


where $R_{\alpha}^{i \dagger}$ is the operator adjoint to $R_{\alpha}^{i}$ (its precise definition, which includes a sign depending on the Grassmann parity, follows from the formulae).

$\delta$ is a nilpotent antiderivation by (4.5). It therefore establishes the cohomological groups $H_{k}(\delta)$ at antifield number $k$ in the space of local functionals (3.4). By construction, $\delta$ is acyclic at all positive antifield numbers $\left(H_{k}(\delta) \simeq 0 \forall k>0\right)$ when $S$ encodes all the gauge and rigid symmetries (of first and higher order) [8]. In contrast, when only a subset of the rigid symmetries is included, $H_{k}(\delta)$ corresponds at positive antifield number $k$ to the remaining rigid symmetries of order $k$ and is represented by functionals that would be of the form $M^{\hat{a}}(\xi) \delta \xi_{\hat{a}}^{*}$ if all the rigid symmetries had been included. Hence, $H_{1}(\delta)$ is represented by functionals

$$
M^{\hat{a}}(\xi) \int d^{n} x\left(\Delta_{\hat{a}} \phi^{i}\right) \phi_{i}^{*}
$$

\section{Deformation theory}

The extended antifield formalism allows one to describe deformations of a given model and some of its symmetries as deformations of the solution of the extended master equation along the lines of [2]. However, as anticipated in the introduction, a deformation does not necessarily preserve the property that the selected subset of symmetries is a closed one. Therefore, the deformation itself may make it necessary to enlarge the subset of symmetries one has started with. In this section we describe how to cope with this phenomenon within a systematic approach to the deformation problem.

The starting point is a solution $\stackrel{(0)}{S}$ of the extended master equation which encodes the original (undeformed) classical action, its gauge symmetries and a closed subset $\left\{\Delta_{\underline{a}}^{(0)}\right\}$ of its rigid symmetries. The basic idea is to seek a continuous deformation of this solution of the form

$$
S=\stackrel{(0)}{S}+g \stackrel{(1)}{S}+g^{2} \stackrel{(2)}{S}+\ldots
$$

where $g$ is the deformation parameter. This problem is analysed "perturbatively" by expanding $(S, S)=0$ in $g$,

$$
\begin{gathered}
(\stackrel{(0)}{S}, \stackrel{(0)}{S})=0 \\
(\stackrel{(0)}{S}, \stackrel{(1)}{S})=0 \\
(\stackrel{(1)}{S}, \stackrel{(1)}{S})+2(\stackrel{(0)}{S}, \stackrel{(2)}{S})=0
\end{gathered}
$$

(5.2) is satisfied by assumption. In order to discuss the subsequent equations, one may now be tempted to adopt the arguments valid for deformations preserving only the gauge symmetries, as given in [2]. One would then conclude from (5.3) that $\stackrel{(1)}{S}$ must be invariant under the undeformed extended BRST differential $\stackrel{(0)}{s}$, as the latter is 
generated by the antibracket with $\stackrel{(0)}{S}$, see $(4.1)$. Furthermore one can assume without loss of generality that $\stackrel{(1)}{S}$ is nontrivial in the cohomology of $\stackrel{(0)}{S}$, because otherwise it can be removed through local field redefinitions and/or redefinitions of the gauge and rigid symmetry transformations by adding trivial symmetries. This follows from standard arguments which parallel those for deformations of gauge symmetries (see e.g. [3]) and are not repeated here. In this way one would conclude that $\stackrel{(1)}{S}$ represents a nontrivial cohomology class of $H^{0}(\stackrel{(0)}{S})$, the cohomology of $\stackrel{(0)}{S}$ at ghost number 0 in the space of local functionals (3.4). However, this kind of reasoning overlooks that $\stackrel{(0)}{S}$ encodes only a subset of the rigid symmetries and may thus be extended, if necessary.

In order to discuss this possibility, we analyse (5.3) and the subsequent equations more carefully by expanding them in the antifield number. To this end we denote the decomposition of $\stackrel{(n)}{S}$ by

$$
\stackrel{(n)}{S}=\sum_{k \geq 0} \stackrel{(n)}{S}_{k}, \quad \text { agh } \stackrel{(n)}{S_{k}}=k .
$$

The interpretation of the various terms in this expansion follows from the general discussion in section III: $\stackrel{(n)}{S_{0}}$ is the deformation of the original classical action at order $(n)$ $n$ in $g, S_{1}$ encodes the $n$th order deformations of the symmetry transformations under $(n)$

study, $S_{2}$ yields the $n$th order deformation of the graded commutator algebra of these symmetries etc.. Using the expansion of $\stackrel{(0)}{s}$ in the antifield number as in (4.4), Eq. (5.3) decomposes into

$$
\begin{gathered}
\stackrel{(0)}{\gamma} \stackrel{(1)}{S}_{0}+\stackrel{(0)}{\delta} \stackrel{(1)}{S_{1}}=0 \\
\stackrel{(0)}{(0)}_{S_{1}}^{(1)}{ }_{0}+\stackrel{(0)}{\gamma} \stackrel{(1)}{S_{1}}+\stackrel{(0)}{\delta} \stackrel{(1)}{S_{2}}=0
\end{gathered}
$$

(5.6) requires $\stackrel{(1)}{S_{0}}$ to be invariant on-shell under the undeformed gauge and rigid symmetries under study, where "on-shell" refers to the undeformed equations of motion. This is so because the undeformed symmetries under study and the original equations of motion are encoded in $\stackrel{(0)}{\gamma}$ and $\stackrel{(0)}{\delta}$ respectively. Let us assume we have found a solution to (5.6). The possible need for an enhancement of the subset of rigid symmetries under study arises for the first time in the next step, i.e., when seeking a solution of (5.7). To see this we act with $\stackrel{(0)}{\gamma}$ on (5.6). Using the anticommutation relations (4.5) for $\stackrel{(0)}{s}$, we infer that the functional $W_{1}$ defined by

$$
W_{1}=\stackrel{(0)}{s_{1}} \stackrel{(1)}{S_{0}}+\stackrel{(0)}{\gamma} \stackrel{(1)}{S_{1}}
$$


is $\stackrel{(0)}{\delta}$-closed,

$$
\stackrel{(0)}{\delta} W_{1}=0
$$

Now, (5.7) requires that $W_{1}$ be $\stackrel{(0)}{\delta}$-exact. (5.9) is thus a necessary condition for the existence of a solution to (5.7). However, it is not sufficient in general when $\stackrel{(0)}{s}$ encodes only a subset of the rigid symmetries, see section $\mathbb{I V}$.

The question at this stage is therefore: can it happen that $W_{1}$ contains a rigid symmetry of the original action which is not contained in the closed subset of symmetries one has started with? The answer to this question is affirmative, as we shall illustrate explicitly in the next sections. Hence, as $W_{1}$ has antifield number 1 , it may contain contributions of the form (4.7). Furthermore $W_{1}$ has ghost number 1 . Its general form is thus

$$
W_{1}=\frac{1}{2}(-)^{\epsilon_{\underline{a}}}+\epsilon_{\hat{c}} f_{\underline{a b}}^{\hat{c}} \xi^{\underline{b}} \xi \underline{\underline{a}} \int d^{n} x\left(\stackrel{(0)}{\Delta_{\hat{c}}} \phi^{i}\right) \phi_{i}^{*}-\stackrel{(0)}{\delta}(\ldots) .
$$

Recall that $\stackrel{(0)}{\Delta}_{\hat{c}}$ denotes a rigid symmetry of $\stackrel{(0)}{S_{0}}$ that is not contained in $\left\{\stackrel{(0)}{\Delta_{a}}\right\}$. If such symmetries occur in $W_{1}$, i.e., if there are nonvanishing coefficients $f_{\underline{a b}}^{\hat{c}}$, the subset of rigid symmetries under study needs to be enlarged by including these symmetries in order to sove (5.7). Of course, this requires first of all to construct a new solution $\stackrel{(0)}{S}$ of the extended master equation which incorporates the additional symmetries too, and then to reexamine Eqs. (5.6) and (5.7) as $\stackrel{(0)}{s}$ gets extended.

Let us assume now that Eqs. (5.6) and (5.7) have been solved. Then there are no further obstructions to a solution of Eq. (5.3) if higher order symmetries are absent, i.e., all the equations subsequent to (5.7) can be solved without further ado because

then $\stackrel{(0)}{\delta}$ is acyclic at all antifield numbers exceeding 1 . In contrast, if there are higher order symmetries, it cannot be excluded in principle that some of them show up at a certain stage and must be included too.

Once one has solved (5.3), one has to analyse (5.4) and the subsequent equations. Now, one has $(\stackrel{(0)}{S},(\stackrel{(1)}{S}, \stackrel{(1)}{S}))=0$ as a consequence of (5.3), thanks to the Jacobi identity for the extended antibracket. $(\stackrel{(1)}{S}, \stackrel{(1)}{S})$ has ghost number 1 and is thus a cocycle in $H^{1}\left(\begin{array}{c}(0) \\ S\end{array}\right)$. This is a necessary condition for the existence of a solution to (5.4) but, in general, it is not sufficient because (5.4) requires that $(\stackrel{(1)}{S}, \stackrel{(1)}{S})$ be $\stackrel{(0)}{S}$ exact. Therefore (5.4) may obstruct deformations through $H^{1}(\stackrel{(0)}{S})$. Note however that some of the cohomology classes in $H^{1}(\stackrel{(0)}{S})$ will originate from rigid symmetries that have not been included so far. These classes are represented by $\stackrel{(0)}{s}$-invariant extensions of functionals of the form (4.7) and their analogues for higher order rigid symmetries (if any). Such classes can be removed by further extending the subset 
of rigid symmetries. We shall therefore refer to them as "spurious anomalies", and call the other classes "true anomalies". p These two kinds of anomalies show up at different antifield numbers 9 . Using the expansion

$$
(\stackrel{(1)}{S}, \stackrel{(1)}{S})=-2 \sum_{k \geq 0} A_{k} \quad, \quad \operatorname{agh} A_{k}=k,
$$

(5.4) decomposes into

$$
\begin{gathered}
A_{0}=\stackrel{(0)}{\gamma} \stackrel{(2)}{S_{0}}+\stackrel{(0)}{\delta} \stackrel{(2)}{S_{1}} \\
A_{1}=\stackrel{(0)}{S_{1}} \stackrel{(2)}{S_{0}}+\stackrel{(0)}{\gamma} \stackrel{(2)}{S_{1}}+\stackrel{(0)}{\delta} \stackrel{(2)}{S_{0}}
\end{gathered}
$$

True anomalies can show up only in $A_{0}$ through contributions that are weakly (= onshell) $\stackrel{(0)}{\gamma}$-closed but not weakly $\stackrel{(0)}{\gamma}$-exact. They can thus obstruct (5.12). In contrast, spurious anomalies would show up in the $A_{k}$ with $k>0$, and thus in (5.13) and the equations subsequent to it. Thereby spurious anomalies stemming from rigid symmetries of order $k$ would show up in $A_{k}$. In particular, when higher order rigid symmetries are absent, actually only $A_{1}$ can give rise to spurious anomalies through terms of the form (4.7) with ghost number 1 . Analogously one analyses the equations subsequent to (5.4) and infers that they can obstruct the deformation in the same way through $H^{1}(\stackrel{(0)}{s})$.

To summarize, the extended antifield formalism permits a systematic analysis of deformations preserving certain rigid symmetries in addition to the gauge symmetries in a manner which is quite similar to the deformation theory [2] based on the standard antifield formalism. The main difference is that the deformation itself may force one to enlarge the subset of rigid symmetries one has started with. It should be clear from the above discussion that in general one cannot predict from the outset which symmetries need to be included in addition to those one has started with because that may depend on the solution to (5.6).

A deformation which requires the enlargement of an originally closed subset of symmetries results in a deformed symmetry algebra. For instance, (5.10) would yield

$$
\stackrel{(1)}{S_{2}}=\frac{1}{2}(-)^{\epsilon_{\underline{b}}}+1 \xi^{\underline{b}} \underline{\xi} \underline{\underline{a}} \stackrel{(1)}{f_{\underline{a b}}^{\hat{c}}} \xi_{\hat{c}}^{*}+\ldots
$$

\footnotetext{
"The term "anomaly" is (ab)used here because these obstructions parallel those to the SlavnovTaylor identity through gauge anomalies in quantum field theory. Indeed, the Slavnov-Taylor identity can be cast in the form of the master equation [19, 20 and the gauge anomalies represent BRST cohomology classes at ghost number 1 [21].

${ }^{6}$ I have not found an example where $(\stackrel{(1)}{S}, \stackrel{(1)}{S})$ contains spurious anomalies. On the other hand, I have neither found a general argument which excludes the occurrence of spurious anomalies. Hence, the question whether or not such anomalies can really occur in $(\stackrel{(1)}{S}, \stackrel{(1)}{S})$ is actually still open.
} 
This shows that the graded commutator algebra of the $\Delta_{\underline{a}}$ (i.e., of the deformed transformations) would not close anymore in the soft sense but involve the $\Delta_{\hat{c}}$. The (1)

$f_{\underline{a b}}^{\hat{c}}$ are the corresponding structure constants of the deformed graded Lie algebra to first order.

\section{Central charge of the $\mathrm{N}=2$ hypermultiplet}

As an illustration, we shall now treat an $\mathrm{N}=2$ supersymmetric model for a FayetSohnius hypermultiplet [11, 12] in flat four dimensional spacetime. The multiplet contains two complex Lorentz-scalar fields $\varphi^{i}(i=1,2)$ and two complex Weyl-spinor fields $\left.\chi^{\alpha}, \psi^{\alpha}(\alpha=1,2)\right]$. As basis of the classical fields we use these fields and their complex conjugates (equivalently we could have chosen for instance the real and imaginary parts of the fields),

$$
\{\phi\}=\left\{\varphi^{i}, \bar{\varphi}_{i}, \chi^{\alpha}, \bar{\chi}^{\dot{\alpha}}, \psi^{\alpha}, \bar{\psi}^{\dot{\alpha}}\right\}
$$

where $\bar{\varphi}_{i}, \bar{\chi}$ and $\bar{\psi}$ are complex conjugate to $\varphi^{i}, \chi$ and $\psi$ respectively,

$$
\bar{\varphi}_{i}=\overline{\varphi^{i}}, \quad \bar{\chi}^{\dot{\alpha}}=\overline{\chi^{\alpha}}, \quad \bar{\psi}^{\dot{\alpha}}=\overline{\psi^{\alpha}} .
$$

The position of the index of $\bar{\varphi}_{i}$ indicates that it transforms contragrediently to $\varphi^{i}$ under the $\mathrm{SU}(2)$-automorphism group of $\mathrm{N}=2$ supersymmetry ( $i$ refers to the fundamental representation of this $\mathrm{SU}(2))$. Undotted and dotted spinor indices distinguish the $(1 / 2,0)$ and $(0,1 / 2)$ representations of the Lorentz group (resp., of its covering group $\mathrm{SL}(2, \mathrm{C}))$.

Our starting point is the action

$$
\stackrel{(0)}{S}_{0}=\int d^{4} x\left[\partial_{\mu} \varphi^{i} \partial^{\mu} \bar{\varphi}_{i}-\frac{\mathrm{i}}{2}(\chi \not \partial \bar{\chi}+\bar{\chi} \not \partial \chi+\psi \not \partial \bar{\psi}+\bar{\psi} \not \partial \psi)\right]
$$

where

$$
\not_{\alpha \dot{\alpha}}=\sigma_{\alpha \dot{\alpha}}^{\mu} \partial_{\mu}
$$

(0)

The action $S_{0}$ is among others invariant under rigid $\mathrm{N}=2$ supersymmetry transfor(0) (0) mations $\Delta_{\alpha}^{i}, \Delta_{\alpha i}$ given by

\begin{tabular}{c|cccccc}
$\phi$ & $\varphi^{j}$ & $\bar{\varphi}_{j}$ & $\chi^{\beta}$ & $\bar{\chi}^{\dot{\beta}}$ & $\psi^{\beta}$ & $\bar{\psi}^{\dot{\beta}}$ \\
\hline${\stackrel{(0)}{\Delta}{ }_{\alpha}{ }^{i} \phi}^{i j} \epsilon^{i j} \chi_{\alpha}$ & $\delta_{j}^{i} \psi_{\alpha}$ & 0 & $-\mathrm{i} \partial_{\alpha}^{\dot{\beta}} \bar{\varphi}^{i}$ & 0 & $-\mathrm{i} \not_{\alpha}^{\dot{\beta}} \varphi^{i}$ \\
\hline$\stackrel{(0)}{\Delta}_{\alpha i} \phi$ & $\delta_{i}^{j} \bar{\psi}_{\dot{\alpha}}$ & $-\epsilon_{i j} \bar{\chi}_{\dot{\alpha}}$ & $\mathrm{i} \not_{\dot{\alpha}}^{\beta} \varphi_{i}$ & 0 & $-\mathrm{i} \not_{\dot{\alpha}}^{\beta} \bar{\varphi}_{i}$ & 0
\end{tabular}

\footnotetext{
${ }^{7}$ We use conventions with a Minkowski metric $\eta_{\mu \nu}=\operatorname{diag}(+,-,-,-)$ as in 22] which differ only through signs from those in $[16$.
} 
where indices $i$ are raised and lowered with the rules

$$
X^{i}=\epsilon^{i j} X_{j}, X_{i}=\epsilon_{i j} X^{j}, \epsilon^{i j}=-\epsilon^{j i}, \epsilon_{i j}=-\epsilon_{j i}, \epsilon^{12}=\epsilon_{21}=1 .
$$

We consider the following subset of rigid symmetries, containing the supersymmetry transformations and the spacetime translations,

$$
\left\{\stackrel{(0)}{\Delta_{a}}\right\} \equiv\left\{\stackrel{(0)}{\Delta}_{\alpha}^{i}, \stackrel{(0)}{\Delta_{\alpha} i}, \partial_{\mu}\right\}
$$

The graded commutator algebra of these symmetries reads

$$
\left[\stackrel{(0)}{\Delta}_{\alpha}^{i}, \stackrel{(0)}{\Delta}_{\dot{\alpha} j}\right] \approx-\mathrm{i} \delta_{j}^{i} \partial_{\alpha \dot{\alpha}} \quad, \quad\left[\stackrel{(0)}{\Delta}_{\underline{a}}, \stackrel{(0)}{\Delta}_{\underline{b}}\right] \approx 0 \text { otherwise }
$$

where $\approx$ denotes equality up to on-shell trivial symmetries. (6.4) is indeed the $\mathrm{N}=2$ supersymmetry algebra without central charge (on-shell). The action has no gauge symmetries. Therefore it has no higher order symmetries either [23]. This implies the existence of a solution to the extended master equation which encodes only the symmetries (6.3) and their graded commutator algebra. This solution, which was computed first in [24], reads

$$
\begin{aligned}
\stackrel{(0)}{S}= & \stackrel{(0)}{S_{0}}+\stackrel{(0)}{S_{1}}+\stackrel{(0)}{S_{2}} \\
\stackrel{(0)}{S_{1}}= & -\int d^{4} x \sum_{\phi}\left(\xi_{i}^{\alpha} \Delta_{\alpha}^{(0)}{ }^{i} \phi+\bar{\xi}^{\dot{\alpha} i} \stackrel{(0)}{\Delta}_{\dot{\alpha} i} \phi+\xi^{\mu} \partial_{\mu} \phi\right) \phi^{*} \\
\stackrel{(0)}{S}_{2}= & -\mathrm{i} \xi_{i} \sigma^{\mu} \bar{\xi}^{i} \xi_{\mu}^{*}+\int d^{4} x\left[\bar{\chi}^{*} \bar{\xi}^{i} \xi_{i} \chi^{*}+\bar{\psi}^{*} \bar{\xi}^{i} \xi_{i} \psi^{*}\right. \\
& \left.+\frac{1}{2} \xi_{i} \xi^{i} \bar{\chi}^{*} \bar{\psi}^{*}+\frac{1}{2} \bar{\xi}^{i} \bar{\xi}_{i} \psi^{*} \chi^{*}\right]
\end{aligned}
$$

where

$$
\left\{\phi^{*}\right\}=\left\{\varphi_{i}^{*}, \bar{\varphi}^{i *}, \chi_{\alpha}^{*}, \bar{\chi}_{\dot{\alpha}}^{*}, \psi_{\alpha}^{*}, \bar{\psi}_{\dot{\alpha}}^{*}\right\} .
$$

The supersymmetry ghosts $\xi_{i}^{\alpha}$ and $\bar{\xi}^{\dot{\alpha} i}$ are Grassmann even and the translation ghosts $\xi^{\mu}$ are Grassmann odd. The ghosts and antifields have the reality properties

$$
\begin{gathered}
\bar{\xi}^{\dot{\alpha} i}=\overline{\xi_{i}^{\alpha}} \quad, \quad \xi^{\mu}=\overline{\xi^{\mu}} \quad, \quad \bar{\xi}_{\dot{\alpha} i}^{*}=-\overline{\xi_{\alpha}^{i *}}, \quad \xi_{\mu}^{*}=-\overline{\xi_{\mu}^{*}} \\
\bar{\varphi}^{i *}=-\overline{\varphi_{i}^{*}} \quad, \quad \bar{\chi}_{\dot{\alpha}}^{*}=-\overline{\chi_{\alpha}^{*}} \quad, \quad \bar{\psi}_{\dot{\alpha}}^{*}=-\overline{\psi_{\alpha}^{*}} .
\end{gathered}
$$

The first term in $\stackrel{(0)}{S_{2}}$ contains the structure constants of the supersymmetry algebra (6.4), while the contributions which are quadratic in the antifields reflect that the symmetry algebra closes only on-shell.

We now study deformations of the above model along the lines of the previous section. A solution to (5.6) which introduces mass terms for the fermions is easily found. Namely,

$$
\stackrel{(1)}{S_{0}}=\int d^{4} x\left[m_{1} \chi \psi+\bar{m}_{1} \bar{\chi} \bar{\psi}+\frac{1}{2}\left(m_{2} \chi \chi+\bar{m}_{2} \bar{\chi} \bar{\chi}+m_{3} \psi \psi+\bar{m}_{3} \bar{\psi} \bar{\psi}\right)\right]
$$


is supersymmetric on-shell and translation invariant for any choice of complex mass parameters $m_{1}, m_{2}$ and $m_{3}$ and therefore yields a solution to (5.6). The corresponding functional $\stackrel{(1)}{S}$ is

$$
\begin{aligned}
\stackrel{(1)}{S_{1}}=\int & d^{4} x\left[m_{1}\left(\bar{\varphi}_{i} \bar{\chi}^{*} \bar{\xi}^{i}-\varphi_{i} \bar{\psi}^{*} \bar{\xi}^{i}\right)-\bar{m}_{1}\left(\bar{\varphi}^{i} \xi_{i} \psi^{*}+\varphi^{i} \xi_{i} \chi^{*}\right)\right. \\
& \left.-m_{2} \varphi_{i} \bar{\chi}^{*} \bar{\xi}^{i}-\bar{m}_{2} \bar{\varphi}^{i} \xi_{i} \chi^{*}+m_{3} \bar{\varphi}_{i} \bar{\psi}^{*} \bar{\xi}^{i}-\bar{m}_{3} \varphi^{i} \xi_{i} \psi^{*}\right] .
\end{aligned}
$$

Next we calculate the functional $W_{1}$ in (5.8). The result is

$$
\begin{array}{r}
\stackrel{(0)}{s_{1}} \stackrel{(1)}{S_{0}}+\stackrel{(0)}{\gamma} \stackrel{(1)}{S_{1}}=\frac{1}{2} \bar{\xi}^{i} \bar{\xi}_{i} \int d^{4} x\left[\left(-m_{1} \varphi^{j}+m_{3} \bar{\varphi}^{j}\right) \varphi_{j}^{*}+\bar{\varphi}^{j *}\left(m_{1} \bar{\varphi}_{j}-m_{2} \varphi_{j}\right)\right. \\
-\left(m_{1} \chi+m_{3} \psi\right) \chi^{*}+\bar{\chi}^{*}\left(m_{1} \bar{\chi}-m_{2} \bar{\psi}\right) \\
\left.+\left(m_{1} \psi+m_{2} \chi\right) \psi^{*}+\bar{\psi}^{*}\left(-m_{1} \bar{\psi}+m_{3} \bar{\chi}\right)\right]+ \text { c.c. }
\end{array}
$$

where c.c. denotes complex conjugation. (6.8) has the form of the first term in (5.10), i.e., it brings in an additional symmetry. This symmetry is part of a rigid SU(2)invariance of the action (6.1). Indeed, as the functional (6.8) is ${ }^{(0)} \delta$-invariant for any choice of $m_{1}, m_{2}$ and $m_{3}$, the parts in (6.8) involving $m_{1}, m_{2}$ and $m_{3}$ respectively correspond to independent symmetries of the action (6.1). These symmetries form an $\mathrm{SU}(2)$ under which $\left(\varphi^{1}, \bar{\varphi}^{1}\right),\left(\varphi^{2}, \bar{\varphi}^{2}\right),(\chi, \psi)$ and $(\bar{\psi}, \bar{\chi})$ transform as doublets (i.e., in the fundamental representation) and which commutes with the supersymmetry transformations (6.2). However, in contrast to the undeformed action, the first order deformation (6.6) is not invariant under the full $\mathrm{SU}(2)$ but it is still invariant under a $U(1)$ subgroup thereof generated by the transformations in (6.8). Hence, the deformation breaks the $\mathrm{SU}(2)$ but preserves this $\mathrm{U}(1)$ subgroup 8 .

We thus have to enlarge the subset of symmetries (6.3) by this $\mathrm{U}(1)$. It turns out that this suffices in order to construct a deformed solution of the extended master equation. We shall not further discuss the computation and spell out the solution only for the case $m_{2}=m_{3}=0$. Using $m=g m_{1}$ ( $g$ being the deformation parameter in the notation of the previous section), the deformed solution reads then

$$
\begin{aligned}
S= & S_{0}+S_{1}+S_{2} \\
S_{0}= & \stackrel{(0)}{0}_{0}+\int d^{4} x\left(m \chi \psi+\bar{m} \bar{\chi} \bar{\psi}-m \bar{m} \varphi^{i} \bar{\varphi}_{i}\right) \\
S_{1}= & \int d^{4} x\left[-\sum_{\phi}\left(\xi_{i}^{\alpha} \Delta_{\alpha}^{(0)} \phi+\bar{\xi}^{\dot{\alpha} i} \Delta_{\alpha i}^{(0)} \phi+\xi^{\mu} \partial_{\mu} \phi\right) \phi^{*}\right. \\
& +\mathrm{i} \xi_{U(1)}\left(\varphi^{i} \varphi_{i}^{*}-\bar{\varphi}_{i} \bar{\varphi}^{i *}+\chi \chi^{*}-\bar{\chi}^{*} \bar{\chi}-\psi \psi^{*}+\bar{\psi}^{*} \bar{\psi}\right) \\
& \left.+m\left(\bar{\varphi}_{i} \bar{\chi}^{*} \bar{\xi}^{i}-\varphi_{i} \bar{\psi}^{*} \bar{\xi}^{i}\right)-\bar{m}\left(\bar{\varphi}^{i} \xi_{i} \psi^{*}+\varphi^{i} \xi_{i} \chi^{*}\right)\right] \\
& \stackrel{(0)}{S_{2}}+\frac{\mathrm{i}}{2}\left(m \bar{\xi}^{i} \bar{\xi}_{i}+\bar{m} \xi_{i} \xi^{i}\right) \xi_{U(1)}^{*}
\end{aligned}
$$

\footnotetext{
${ }^{8} \mathrm{An}$ analogous phenomenon was observed in 25] within the construction of supergravity couplings for hypermultiplets.
} 
(0)

with $S_{2}$ as in (6.5). Here $\xi_{U(1)}$ and $\xi_{U(1)}^{*}$ are the global ghost and antifield of the rigid $\mathrm{U}(1)$ symmetry obtained from (6.8) in the case $m_{2}=m_{3}=0\left(\xi_{U(1)}\right.$ is real and Grassmann odd, $\xi_{U(1)}^{*}$ is purely imaginary and Grassmann even).

(6.10) is the deformed classical action. Apart from the original action (6.1) and its first order deformation (6.6) (in the case $m_{2}=m_{3}=0$ ), it contains also a mass term for the Lorentz-scalar fields which arises at second order in the deformation parameter.

(6.11) contains the deformed supersymmetry transformations, the rigid $\mathrm{U}(1)$ transformations, and the spacetime translations. The deformed supersymmetry and the $\mathrm{U}(1)$ transformations are

\begin{tabular}{c|cccccc}
$\phi$ & $\varphi^{j}$ & $\bar{\varphi}_{j}$ & $\chi^{\beta}$ & $\bar{\chi}^{\dot{\beta}}$ & $\psi^{\beta}$ & $\bar{\psi}^{\dot{\beta}}$ \\
\hline$\Delta_{\alpha}{ }^{i} \phi$ & $\epsilon^{i j} \chi_{\alpha}$ & $\delta_{j}^{i} \psi_{\alpha}$ & $\bar{m} \delta_{\alpha}^{\beta} \varphi^{i}$ & $-\mathrm{i} \partial_{\alpha}^{\dot{\beta}} \bar{\varphi}^{i}$ & $\bar{m} \delta_{\alpha}^{\beta} \bar{\varphi}^{i}$ & $-\mathrm{i} \partial_{\alpha}^{\dot{\beta}} \varphi^{i}$ \\
\hline$\Delta_{\dot{\alpha} i} \phi$ & $\delta_{i}^{j} \bar{\psi}_{\dot{\alpha}}$ & $-\epsilon_{i j} \bar{\chi}_{\dot{\alpha}}$ & $\mathrm{i} \partial_{\dot{\alpha}}^{\beta} \varphi_{i}$ & $-m \delta_{\dot{\alpha}}^{\dot{\beta}} \bar{\varphi}_{i}$ & $-\mathrm{i} \not_{\dot{\alpha}}^{\beta} \bar{\varphi}_{i}$ & $m \delta_{\dot{\alpha}}^{\dot{\beta}} \varphi_{i}$ \\
\hline$\Delta_{U(1)} \phi$ & $-\mathrm{i} \varphi^{j}$ & $\mathrm{i} \bar{\varphi}_{j}$ & $-\mathrm{i} \chi^{\beta}$ & $\mathrm{i} \bar{\chi}^{\dot{\beta}}$ & $\mathrm{i} \psi^{\beta}$ & $-\mathrm{i} \bar{\psi}^{\dot{\beta}}$
\end{tabular}

(6.12) encodes the graded commutator algebra of the deformed symmetry transformations. The $\mathrm{N}=2$ supersymmetry algebra has become extended by the $\mathrm{U}(1)$ through the deformation. The nonvanishing graded commutators are

$$
\begin{aligned}
{\left[\Delta_{\alpha}{ }^{i}, \Delta_{\dot{\alpha} j}\right] } & \approx-\mathrm{i} \delta_{j}^{i} \partial_{\alpha \dot{\alpha}} \\
{\left[\Delta_{\alpha}{ }^{i}, \Delta_{\beta}{ }^{j}\right] } & \approx \mathrm{i} \bar{m} \epsilon^{i j} \epsilon_{\alpha \beta} \Delta_{U(1)} \\
{\left[\Delta_{\dot{\alpha} i}, \Delta_{\dot{\beta} j}\right] } & \approx-\mathrm{i} m \epsilon_{i j} \epsilon_{\dot{\alpha} \dot{\beta}} \Delta_{U(1)}
\end{aligned}
$$

where $\approx$ now denotes equality up to transformations which are trivial on-shell in the deformed model (i.e., these transformations involve the deformed equations of motion).

REMARK. The above results hold analogously in a formulation of the hypermultiplet with the standard auxiliary fields used already in [11, 12]. In that approach one sometimes introduces an "off-shell central charge" in order to close the commutator algebra of the supersymmetries, the central charge and the spacetime translations off-shell. However, in the massless model that central charge is trivial on-shell and thus not to be accompanied by global ghosts. In contrast, the massive (deformed) model involves again a "true" central charge that does not vanish on-shell.

\section{Curci-Ferrari model}

A particular case of a rigid symmetry is the BRST symmetry of a gauge fixed action constructed in the standard way from a solution to the usual master equation [5, 6, 7]. Deformations of a gauge fixed action may be obtained in two ways: (i) one constructs first consistent deformations of the underlying gauge theory along the lines of [2] and 
fixes the gauge afterwards, or (ii) one investigates directly deformations of the gauge fixed model and its BRST symmetry.

These two approaches are not equivalent in general. In particular, the first approach leads by construction to an on-shell nilpotent BRST symmetry of the standard type, whereas the second one may destroy the nilpotency property and is not physically acceptable in general. This is related to the different properties of the BRST cohomology before and after gauge fixing [26] (cf. also the remark at the end of this section), and is now to be discussed for the Curci-Ferrari model [13, 14, 15] in the framework of the extended antifield formalism. The loss of nilpotency emerges in this approach as a deformation of the BRST algebra along the lines of section $\mathrm{V}$ : in this particular case, the deformed action has even the same BRST symmetry as the original one, but in the deformed model that symmetry does not square weakly to zero anymore (as the equations of motion change). Rather, it squares into a different nontrivial rigid symmetry.

We consider four dimensional nonabelian Yang-Mills theory with the following gauge fixed action?,

$$
\stackrel{(0)}{S_{0}}=\int d^{4} x \operatorname{Tr}\left[\frac{1}{4} F_{\mu \nu} F^{\mu \nu}+\frac{1}{2 \alpha}\left(\partial_{\mu} A^{\mu}\right)^{2}-\frac{1}{2} B\left(\partial_{\mu} D^{\mu}+D_{\mu} \partial^{\mu}\right) C+\frac{\alpha e^{2}}{4} B^{2} C^{2}\right]
$$

where $\alpha$ is the gauge fixing parameter, $e$ is the gauge coupling constant, and $A_{\mu}=$ $A_{\mu}^{i} t_{i}, F_{\mu \nu}=F_{\mu \nu}^{i} t_{i}, C=C^{i} t_{i}$ and $B=B^{i} t_{i}$ are the Lie algebra valued gauge fields, field strengths, ghost fields and antighost fields respectively $\left(\left\{t_{i}\right\}\right.$ denotes an appropriate matrix representation of the Lie algebra of the gauge group normalized such that $\left.\operatorname{Tr}\left(t_{i} t_{j}\right)=-\delta_{i j}\right)$, and $D_{\mu} C$ is defined by

$$
D_{\mu} C=\partial_{\mu} C+e\left(A_{\mu} C-C A_{\mu}\right) .
$$

The action (7.1) is invariant under the rigid BRST transformations

$$
\Delta_{\mathrm{brs}} A_{\mu}=D_{\mu} C, \quad \Delta_{\mathrm{brs}} C=-e C^{2}, \quad \Delta_{\mathrm{brs}} B=\frac{1}{\alpha} \partial_{\mu} A^{\mu}-\frac{e}{2}(B C+C B) .
$$

These transformations are nilpotent on-shell,

$$
\left(\Delta_{\mathrm{brs}}\right)^{2}=\frac{1}{2}\left[\Delta_{\mathrm{brs}}, \Delta_{\mathrm{brs}}\right] \approx 0
$$

More precisely, $\Delta_{\mathrm{brs}}$ is strictly nilpotent on $A_{\mu}^{i}$ and $C^{i}$, but squares into an on-shell trivial symmetry on $B^{i}$,

$$
\left(\Delta_{\mathrm{brs}}\right)^{2} A_{\mu}^{i}=\left(\Delta_{\mathrm{brs}}\right)^{2} C^{i}=0, \quad\left(\Delta_{\mathrm{brs}}\right)^{2} B^{i}=\frac{1}{\alpha} \delta^{i j} \frac{\delta^{L} \stackrel{(0)}{S_{0}}}{\delta B^{j}} .
$$

\footnotetext{
${ }^{9}$ The gauge fixed action $(7.1)$ arises in the standard manner from a "minimal" solution $S_{\min }$ of the usual master equation as follows. First one adds to $S_{\min }$ the "nonminimal" $\operatorname{term} \int d^{4} x \operatorname{Tr}\left(-H B^{*}\right)$ where the $H^{i}$ are Nakanishi-Lautrup auxiliary fields. Then one shifts the antifields by $\Phi_{A}^{*} \rightarrow$ $\Phi_{A}^{*}+\delta^{L} \Psi / \delta \Phi^{A}$ where $\Psi$ is the "gauge fixing fermion" $\Psi[\Phi]=\int d^{4} x \operatorname{Tr}\left[(\alpha / 2)\left(B H+e B^{2} C\right)-B \partial_{\mu} A^{\mu}\right]$. Finally one eliminates the $H^{i}$ by their algebraic equations of motion.
} 
We shall now apply the extended antifield formalism to the gauge fixed action (7.1) and the BRST symmetry (7.3). In this approach (7.1) plays the role of the classical action, i.e. the ghost and antighost fields $C$ and $B$ are viewed as Grassmann odd "classical" fields ( $C$ is real, $B$ purely imaginary),

$$
\{\phi\}=\left\{A_{\mu}^{i}, B^{i}, C^{i}\right\}
$$

Accordingly, we assign antifield number 1 to $A_{i}^{\mu *}, B_{i}^{*}$ and $C_{i}^{*}$. Furthermore we introduce a Grassmann even global ghost $\xi_{\text {brs }}$ for the BRST symmetry (7.3).

That is, in this case we consider a subset of rigid symmetries containing only one element, namely $\Delta_{\text {brs }}$ :

$$
\left\{\stackrel{(0)}{\Delta_{a}}\right\}=\left\{\Delta_{\mathrm{brs}}\right\}
$$

The corresponding graded commutator algebra (2.13) is just (7.5). As the gauge fixed action (7.1) has no gauge symmetry and thus no higher order rigid symmetry either, a corresponding solution of the extended master equation exists. This solution coincides of course with the gauge fixed solution of the master equation obtained in the standard antifield formalism, except that now the global ghost $\xi_{\text {brs }}$ appears,

$$
\begin{aligned}
& \stackrel{(0)}{S}=\stackrel{(0)}{S_{0}}+\stackrel{(0)}{S_{1}}+\stackrel{(0)}{S_{2}} \\
& \stackrel{(0)}{S_{1}}=\xi_{\text {brs }} \int d^{4} x \operatorname{Tr}\left[A^{\mu *} D_{\mu} C+e C^{2} C^{*}-\left\{\frac{1}{\alpha} \partial_{\mu} A^{\mu}-\frac{e}{2}(B C+C B)\right\} B^{*}\right] \\
& \stackrel{(0)}{S_{2}}=\frac{1}{2 \alpha} \xi_{\text {brs }}^{2} \int d^{4} x \operatorname{Tr}\left(B^{*} B^{*}\right)
\end{aligned}
$$

where we have used $A^{\mu *}=-\delta^{i j} A_{i}^{\mu *} t_{j}$ etc.. The presence of the term quadratic in $B^{*}$ reflects that the algebra closes on $B^{i}$ only on-shell, see (7.5). The "extended" BRST differential $\stackrel{(0)}{s}$, constructed from $\stackrel{(0)}{S}$ as in Eq. (4.1), coincides with the usual gauge fixed BRST operator for the action (7.1), except that now $\xi_{\text {brs }}$ occurs. It is strictly nilpotent, in contrast to $\Delta_{\text {brs }}$, and acts on the fields by

$$
\begin{aligned}
\stackrel{(0)}{s} A_{\mu} & =\xi_{\mathrm{brs}} D_{\mu} C, \stackrel{(0)}{s} C=-\xi_{\mathrm{brs}} e C^{2} \\
\stackrel{(0)}{s} B & =\xi_{\mathrm{brs}}\left[\frac{1}{\alpha} \partial_{\mu} A^{\mu}-\frac{e}{2}(B C+C B)\right]-\frac{1}{\alpha} \xi_{\mathrm{brs}}^{2} B^{*} .
\end{aligned}
$$

We shall now discuss the deformation of the action (7.1) through the Curci-Ferrari mass term

$$
\stackrel{(1)}{S_{0}}=\int d^{4} x \operatorname{Tr}\left[\frac{1}{2} A_{\mu} A^{\mu}+\alpha B C\right] .
$$

This term is off-shell invariant under the transformations (7.3) and thus yields a solution to Eq. (5.6) with

$$
\stackrel{(1)}{S_{1}}=0
$$


The functional $W_{1}$ in Eq. (5.8) reads in this case

$$
\stackrel{(0)}{s_{1}} \stackrel{(1)}{S_{0}}=-\xi_{\mathrm{brs}}^{2} \int d^{4} x \operatorname{Tr}\left(B^{*} C\right) .
$$

This has the form of the first term in Eq. (5.10) and contains an additional nontrivial rigid symmetry of the gauge fixed action (7.1), namely

$$
\Delta_{\text {add }} B=C, \quad \Delta_{\text {add }} A_{\mu}=\Delta_{\text {add }} C=0 .
$$

Hence, in order to construct a deformed solution of the extended master equation (1) with $S_{0}$ as in (7.9), we must include this symmetry. It is straightforward to verify that this yields the following deformed solution of the extended master equation,

$$
\begin{aligned}
S & =S_{0}+S_{1}+S_{2} \\
S_{0} & =\stackrel{(0)}{S_{0}}+g \stackrel{(1)}{S_{0}} \\
S_{1} & =-\int d^{4} x \sum_{\phi}\left(\xi_{\mathrm{brs}} \Delta_{\mathrm{brs}} \phi+\xi_{\mathrm{add}} \Delta_{\mathrm{add}} \phi\right) \phi^{*} \\
S_{2} & =\stackrel{(0)}{S_{2}}+g \xi_{\mathrm{brs}}^{2} \xi_{\text {add }}^{*} .
\end{aligned}
$$

(0)

with $S_{2}$ as in (7.7). The last term in $(7.13)$ reflects that the graded commutator algebra of $\Delta_{\text {brs }}$ and $\Delta_{\text {add }}$ reads in the deformed model

$$
\left(\Delta_{\mathrm{brs}}\right)^{2} \approx g \Delta_{\mathrm{add}} \quad, \quad\left[\Delta_{\mathrm{brs}}, \Delta_{\mathrm{add}}\right]=0
$$

where $\approx$ now denotes on-shell equality in the deformed model, i.e. for the equations of motion following from $\stackrel{(0)}{S_{0}}+g \stackrel{(1)}{S_{0}}$. Notice that $\Delta_{\text {brs }}$ is still a symmetry of the deformed model, without having been deformed. Nevertheless it is not nilpotent anymore on-shell because the equations of motion have changed.

\section{REMARKS.}

a) In order to avoid possible confusion, we stress that the BRST cohomologies before and after gauge fixing are always isomorphic (provided all the antifields are kept). What changes however when the gauge fixed action is treated as a classical one, are the assignments of antifield numbers and the corresponding concept of weak (= on-shell) equality (as the ghost fields count now among the classical fields). As a consequence, it is not true that each local functional with vanishing antifield number which is on-shell $\Delta_{\text {brs }}$-invariant can be extended to a cocycle of $\stackrel{(0)}{s}$ (this is just the phenomenon discussed in [26], but in the language used here). The Curci-Ferrari mass term (7.9) illustrates exactly this phenomenon: it cannot be extended so as to be $\stackrel{(0)}{s}$-closed, although it is $\Delta_{\text {brs }}$-invariant. As a consequence, $\Delta_{\text {brs }}$ is not nilpotent anymore on-shell in the deformed model. 
b) The Curci-Ferrari model illustrates a general fact: a deformation of a gauge fixed action which destroys the on-shell nilpotency of $\Delta_{\text {brs }}$ (or a deformation thereof) cannot reflect a consistent deformation of the gauge symmetry in the sense of [2] because such consistent deformations result by their very construction in an on-shell nilpotent $\Delta_{\text {brs }}$ after gauge fixing.

c) Of course, (7.13) yields via (4.1) a strictly nilpotent operator which incorporates both $\Delta_{\text {brs }}$ and $\Delta_{\text {add }}$. However, this nilpotent operator cannot cure the unitarity problems of the Curci-Ferrari model discussed in [14, 15] because $\Delta_{\text {add }}$ does not impose additional conditions that may select physical states. Indeed, as it is just the square of $\Delta_{\text {brs }}$ (on-shell), a state that is annihilated by $\Delta_{\text {brs }}$ (resp. by its quantum version) is automatically also annihilated by $\Delta_{\text {add }}$. For the same reason, a state that is $\Delta_{\text {add }}$-exact is also in the image of $\Delta_{\text {brs }}$.

\section{Conclusion}

We have outlined how continuous deformations of an action functional, its gauge symmetries and a closed subset of its rigid symmetries can be analysed systematically in the extended antifield formalism. The procedure is very similar to the study of continuous deformations of actions and their gauge symmetries described in [2]. The main difference is that the deformation itself may make it necessary to enlarge the particular subset of rigid symmetries one has started with. This happens when the commutator algebra of the deformed version of the originally considered subset of symmetries does not close anymore in the soft sense (i.e. modulo gauge transformations and on-shell trivial symmetries) and thus results in a deformation of the symmetry algebra.

It is however not always clear from the outset which additional symmetries can occur in the deformed commutator algebra. This subtlety can be mastered when one proceeds as described in section $\mathrm{Q}$, using an expansion in the antifield number. In this approach one first seeks functionals of the classical fields that are "weakly" (= on-shell) invariant under the symmetries under study. The method then provides automatically the additional symmetries which need to be included. This has been illustrated for the hypermultiplet of four dimensional $\mathrm{N}=2$ supersymmetry where the central extension of the $\mathrm{N}=2$ supersymmetry algebra emerges via the deformation of a massless model to a massive one. The central extension turns out to be a surviving generator of an $\mathrm{SU}(2)$ symmetry of the massless action broken by the deformation. In this case it depends on the mass parameters, i.e. on the deformation itself, how the $\mathrm{SU}(2)$ is broken and which generator becomes the central extension.

We have also illustrated, for the Curci-Ferrari model, how deformations of a gauge fixed action and its BRST symmetry can be analysed within this approach. The BRST symmetry is then treated in the same manner as other rigid symmetries too, while the gauge fixed action is treated as a classical one. However, such deformations do not correspond necessarily to consistent deformations of the gauge symmetries in the sense of [2], and are therefore not always physically acceptable. In particular, 
it can happen that there are deformations of a gauge fixed action which are BRST invariant but nevertheless inconsistent because the BRST symmetry does not square to zero on-shell anymore in the deformed model. The Curci-Ferrari model illustrates exactly this phenomenon. Hence, a necessary condition for a deformation of a gauge fixed action to be a consistent one, is the on-shell nilpotency of the BRST symmetry of the deformed action.

Finally I remark that the procedure outlined in section $\nabla$ can be extended analogously to the case that only a subset of the gauge symmetries is included. However, from the physical point of view this extension is mainly of academic interest and was therefore not discussed here.

\section{Acknowledgements}

Discussions with Tobias Hurth and Kostas Skenderis about the Curci-Ferrari model are gratefully acknowledged. The author was supported by the Deutsche Forschungsgemeinschaft.

\section{References}

[1] M. Gerstenhaber, On the deformation of rings and algebras, Ann. of Math. 79 (1964) 59-103.

[2] G. Barnich and M. Henneaux, Consistent couplings between fields with a gauge freedom and deformations of the master equation, Phys. Lett. B 311 (1993) 123-129 (hep-th/9304057).

[3] M. Henneaux, Consistent interactions between gauge fields: the cohomological approach, to be published in Contemp. Math. (hep-th/9712226).

[4] J.D. Stasheff, Deformation theory and the Batalin-Vilkovisky master equation, in: Deformation Theory and Symplectic Geometry, pp. 271-284, D. Sternheimer et al. (Eds.) (Kluwer Acad. Publ., 1997) (q-alg/9702012).

[5] I.A. Batalin and G.A. Vilkovisky, Gauge algebra and quantization, Phys. Lett. B 102 (1981) 27-31; Quantization of gauge theories with linearly dependent generators, Phys. Rev. D 28 (1983) 2567-2582 (Erratum: ibid. D 30 (1984) $508)$.

[6] M. Henneaux and C. Teitelboim, Quantization of Gauge Systems (Princeton Univ. Press, Princeton, New Jersey, 1992).

[7] J. Gomis, J. París and S. Samuel, Antibracket, antifields and gauge theory quantization, Phys. Rep. 259 (1995) 1-145 (hep-th/9412228). 
[8] F. Brandt, M. Henneaux and A. Wilch, Extended antifield formalism, Nucl. Phys. B 510 (PM) (1998) 640-656 (hep-th/9705007).

[9] F. Brandt, M. Henneaux and A. Wilch, Global symmetries in the antifield formalism, Phys. Lett. B 387 (1996) 320-326 (hep-th/9606172).

[10] R. Haag, J.T. Lopuszanski and M. Sohnius, All possible generators of supersymmetries of the S-matrix, Nucl. Phys. B 88 (1975) 257-274.

[11] P. Fayet, Fermi-Bose hypersymmetry, Nucl. Phys. B 113 (1976) 135-155.

[12] M.F. Sohnius, Supersymmetry and central charges, Nucl. Phys. B 138 (1978) $109-121$.

[13] G. Curci and R. Ferrari, On a class of Lagrangian models for massive and massless Yang-Mills fields, Nuovo Cim. 32A (1976) 151-168; The unitarity problem and the zero-mass limit for a model of massive Yang-Mills theory, ibid. 35A (1976) 1-14.

[14] I. Ojima, Comments on massive and massless Yang-Mills Lagrangians with a quartic coupling of Faddeev-Popov ghosts, Z. Phys. C 13 (1982) 173-177.

[15] J. de Boer, K. Skenderis, P. van Nieuwenhuizen and A. Waldron, On the renormalizability and unitarity of the Curci-Ferrari model for massive vector bosons, Phys. Lett. B 367 (1996) 175-182 (hep-th/9510167).

[16] J. Wess and J. Bagger, Supersymmetry and Supergravity (Princeton Univ. Press, Princeton, New Jersey, 1983).

[17] J.M.L. Fisch and M. Henneaux, Homological perturbation theory and the algebraic structure of the antifield-antibracket formalism for gauge theories, Commun. Math. Phys. 128 (1990) 627-640.

[18] M. Henneaux, Lectures on the antifield-BRST formalism for gauge theories, Nucl. Phys. B (Proc. Suppl.) 18A (1990) 47-106.

[19] J. Zinn-Justin, Renormalization of gauge theories, in: Trends in Elementary Particle Physics, Lecture Notes in Physics 37, pp. 2-39, H. Rollnik and K. Dietz (eds.) (Springer-Verlag, Berlin, 1975).

[20] B.W. Lee, Gauge theories, in: Methods in Field Theory, pp. 79-139, Les Houches 1975, R. Balian and J. Zinn-Justin (eds.) (North-Holland, Amsterdam, 1976).

[21] C. Becchi, A. Rouet and R. Stora, Renormalization of the abelian Higgs-Kibble model, Commun. Math. Phys. 42 (1975) 127-162; Renormalization of gauge theories, Annals Phys. (N.Y.) 98 (1976) 287-321.

[22] F. Brandt, Local BRST cohomology in minimal $D=4, N=1$ supergravity, Annals Phys. (N.Y.) 259 (1997) 253-312 (hep-th/9609192). 
[23] G. Barnich, F. Brandt and M. Henneaux, Local BRST cohomology in the antifield formalism: I. General theorems, Commun. Math. Phys. 174 (1995) 57-91 (hepth/9405109).

[24] L. Baulieu, M. Bellon, S. Ouvry and J.-C. Wallet, Batalin-Vilkovisky analysis of supersymmetric systems, Phys. Lett. B 252 (1990) 387-394.

[25] B. de Wit, J.W. van Holten and A. Van Proeyen, Central charges and conformal supergravity, Phys. Lett. B 95 (1980) 51-55.

[26] M. Henneaux, On the gauge-fixed BRST cohomology, Phys. Lett. B 367 (1996) 163-169 (hep-th/9510116). 